\title{
Large and Versatile Plasmonic Enhancement of Photoluminescence Using Colloidal Metallic Nanocubes
}

\author{
Mohammad Khaywah, ${ }^{1,2}$ Audrey Potdevin, ${ }^{1}$ François Réveret, ${ }^{2}$ Rachid Mahiou, ${ }^{3}$ Youcef Ouerdane, ${ }^{3}$ Anthony \\ Désert, ${ }^{4}$ Stéphane Parola, ${ }^{4}$ Geneviève Chadeyron, ${ }^{1}$ Emmanuel Centeno, ${ }^{2}$ Rafik Smaali, ${ }^{2}$ and Antoine Moreau ${ }^{2}$ \\ ${ }^{1}$ Université Clermont Auvergne, CNRS, SIGMA Clermont, ICCF, 63000 Clermont-Ferrand, France \\ ${ }^{2}$ Université Clermont Auvergne, CNRS, SIGMA Clermont, Institut Pascal, 63000 Clermont-Ferrand, France \\ ${ }^{3}$ Laboratoire Hubert Curien, UMR CNRS 5516, Université Jean Monnet, Saint-Etienne, France \\ ${ }^{4}$ École Normale Supérieure de Lyon, CNRS, Univ. Lyon 1, Laboratoire de Chimie (UMR 5182), Lyon, France
}

\section{CONTENTS}

S1. Nanocube surface density

S2. $\zeta$ potential

S3. Optical response of nanocubes

References

S1. NANOCUBE SURFACE DENSITY
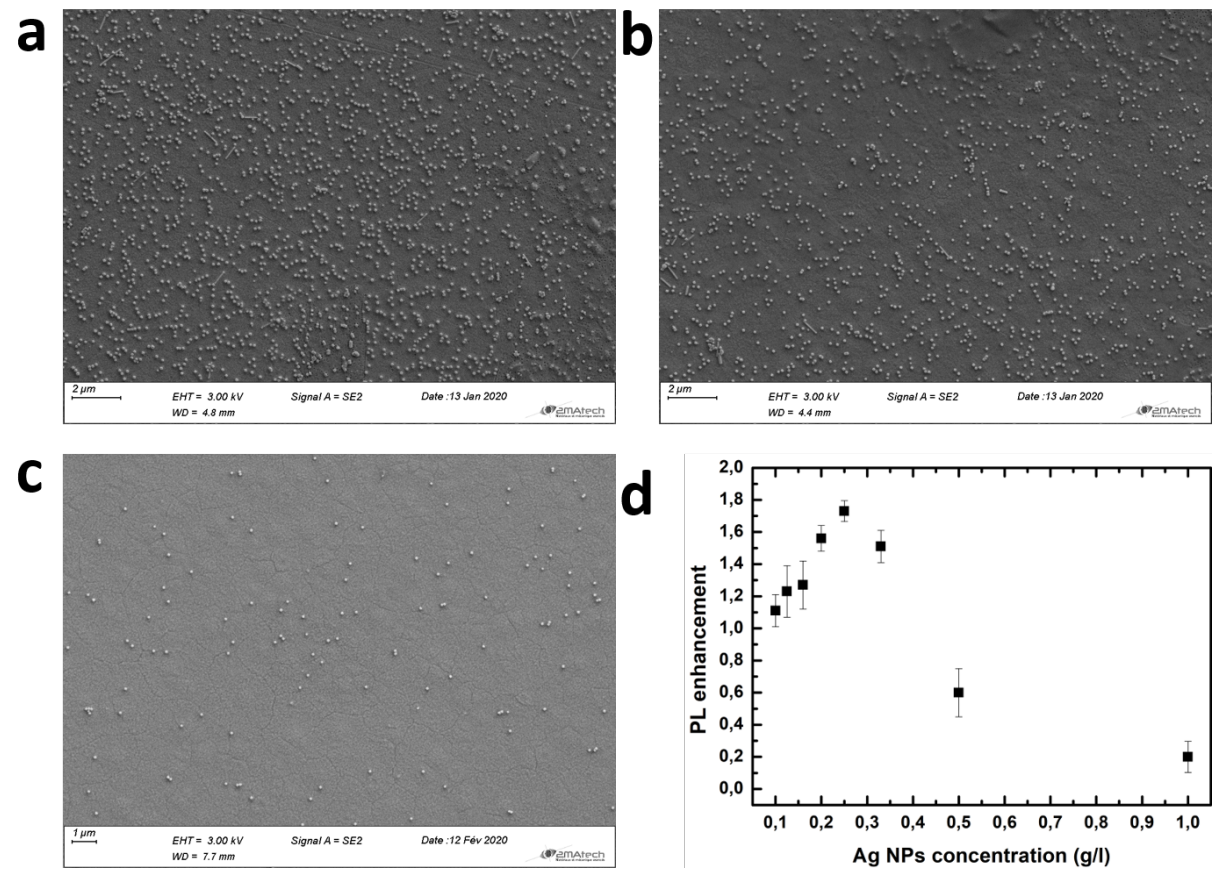

FIG. S1. a, b, c SEM images of the nanocubes deposited on the YAG:Ce layer, allowing to determine the surface density of nanocubes for a given nanoparticle concentration in the solution of respectively $0.5 \mathrm{~g} / 1,0.25 \mathrm{~g} / \mathrm{l}$, and $0.1 \mathrm{~g} / \mathrm{l}$. $\mathbf{d} \mathrm{PL}$ enhancement as a function of the solution concentration which is used for the drop casting. 
S2. $\zeta$ POTENTIAL

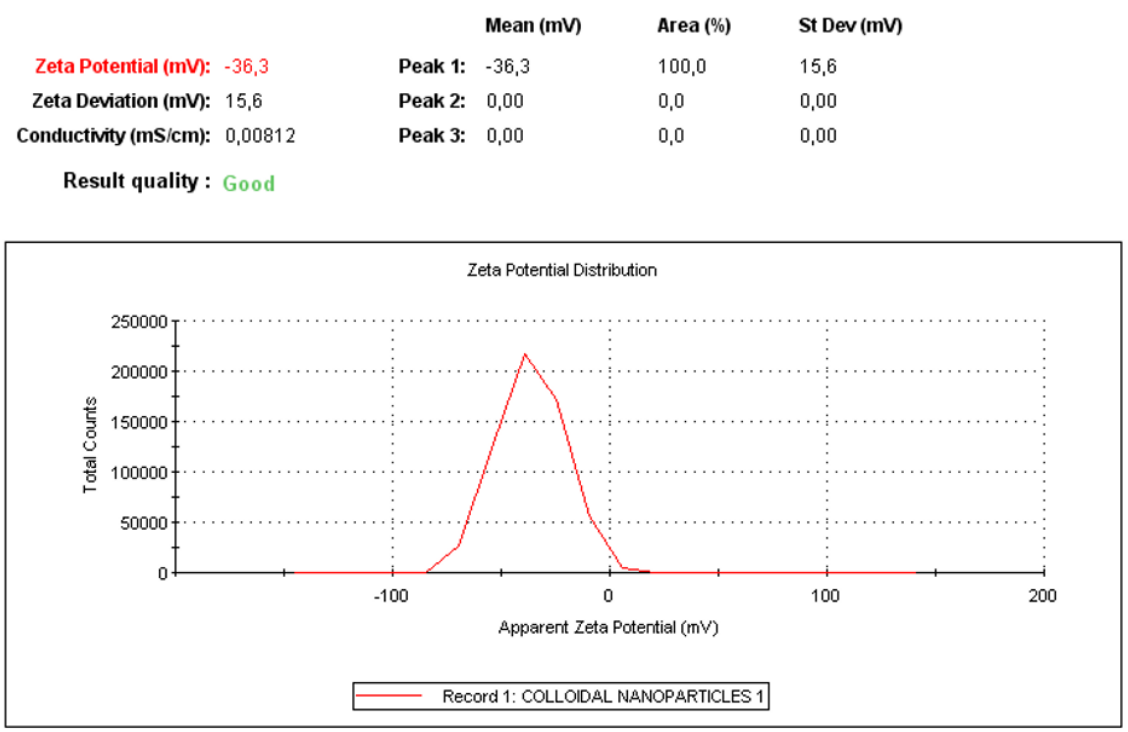

Zeta potentials ( $\zeta$ were measured by laser Doppler electrophoresis (Zetasizer NanoZS, Malvern, UK). The average value of the $\zeta$ potential $(-36.3 \mathrm{mV})$ is characteristic of very stable suspensions [1]. It explains why Ag NCs can be easily spread on a surface with limited aggregation. 


\section{S3. OPTICAL RESPONSE OF NANOCUBES}

In order to better understand the optical response of the nanocubes, we have simulated the interaction of a plane wave with square nanorods deposited on the surface using Fourier Modal Methods[2]. Such 2D simulations represent relatively well the optical behaviour of truly 3D structures[3] because both situations generally share the same resonance mechanism[4]. A way to understand the resonances of nanocubes deposited on a substrate is to map the magnetic field instead of the electric one, as shown in Fig. S1. The resonances on the top and at the bottom of the nanorods clearly appear as cavity resonances for the surface plasmon which propagates at the metal surface. In a cavity, the electric field is strong when there is a node for the magnetic field and vice versa. For small nanocubes, only the fundamental resonances can be excited, leading to enhanced electric fields at the upper and lower nanorod corners only, which is exactly the case for nanocubes[5]. The resonance on top of the nanocubes will allow for a higher reflection of light, while the lower one will allow for a lower reflectance compared to the bare interface. The resonance positions are slightly different because the surface plasmon at the bottom propagates partly in the substrate and thus presents a higher effective index. However, both resonances lead to a larger absorption, as shown in the Figure. The good agreement we observe between our simulations and the experiments for the resonance position in the spectrum strengthens our confidence in our 2D simulations.

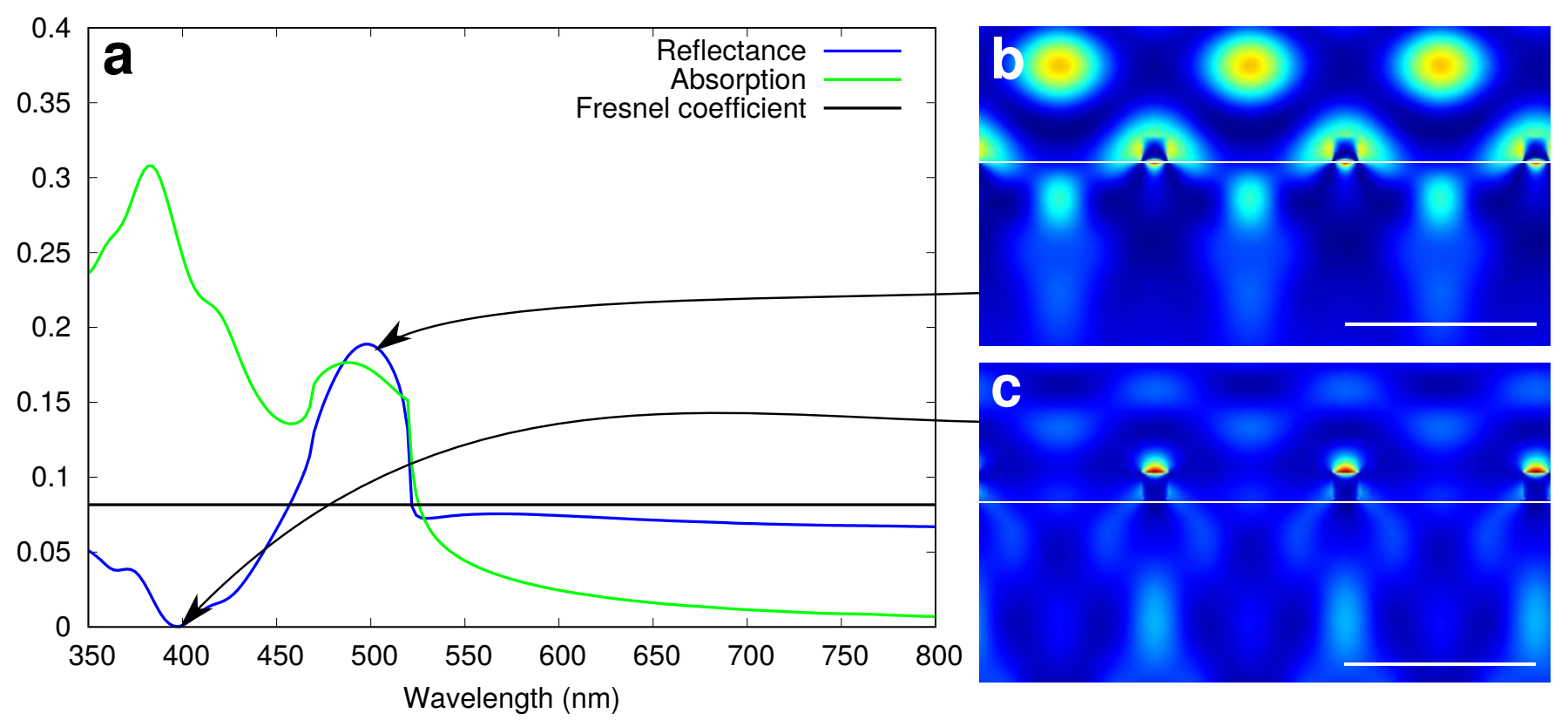

FIG. S2. Nanocubes characterization and simulation. a Specular reflection coefficient in normal incidence with cubes (blue curve) and without (black line) and total absorption (green curve) for $75 \mathrm{~nm}$ wide silver nanocubes periodically (period $d=520 \mathrm{~nm}$ ) deposited on a transparent substrate with a refractive index of 1.8 similar to YAG:Ce. b Magnetic field for a plane wave with a $400 \mathrm{~nm}$ wavelength in vacuum illuminating nanorods placed every $600 \mathrm{~nm}$. c Magnetic field for a wavelength of $500 \mathrm{~nm}$ showing the localized surface plasmon resonance under the nanorods, leading to a higher reflection and absorption.

[1] Vallar, S.; Houivet, D.; El Fallah, J.; Kervadec, D.; Haussonne, J.-M. Oxide slurries stability and powders dispersion: optimization with zeta potential and rheological measurements. Fournal of the European Ceramic Society 1999, 19, 1017-1021.

[2] Granet, G.; Guizal, B. Efficient implementation of the coupled-wave method for metallic lamellar gratings in TM polarization. $70 S A A$ 1996, 13, 1019-1023.

[3] Moreau, A.; Ciracì, C.; Mock, J. J.; Hill, R. T.; Wang, Q.; Wiley, B. J.; Chilkoti, A.; Smith, D. R. Controlled-reflectance surfaces with film-coupled colloidal nanoantennas. Nature 2012, 492, 86-89.

[4] Lemaitre, C.; Centeno, E.; Moreau, A. Interferometric control of the absorption in optical patch antennas. Scientific Reports 2017, 7, 2941.

[5] Zhang, S.; Bao, K.; Halas, N. J.; Xu, H.; Nordlander, P. Substrate-Induced Fano Resonances of a Plasmonic Nanocube: A Route to IncreasedSensitivity Localized Surface Plasmon Resonance Sensors Revealed. Nano Letters 2011, 11, 1657-1663, PMID: 21410217. 